\title{
Network analysis of microRNAs and genes in human osteosarcoma
}

\author{
TIANYAN WANG ${ }^{1,2}$, ZHIWEN XU ${ }^{1-3}$, KUNHAO WANG $^{2,3}$ and NING WANG ${ }^{2,3}$ \\ ${ }^{1}$ College of Software Engineering; ${ }^{2}$ Key Laboratory of Symbolic Computation and Knowledge Engineering \\ of The Ministry of Education; ${ }^{3}$ College of Computer Science and Technology, \\ Jilin University, Changchun, Jilin 130012, P.R. China
}

Received September 16, 2014; Accepted July 27, 2015

DOI: $10.3892 /$ etm.2015.2685

\begin{abstract}
To date, numerous studies have suggested that microRNAs (miRNAs) and genes play key roles in osteosarcoma (OS); however, the majority of these studies have been conducted with a specific focus on either the genes or the miRNAs, which has made the regulatory mechanisms of OS difficult to decipher. The aim of the present study was to systematically investigate the elements [genes, miRNAs and transcription factors (TFs)] associated with the morbidity of OS and to explore the associations among these elements, instead of focusing on one or several elements. The scattered data were collected from existing studies of OS, and three regulatory networks (abnormally expressed, related and global) were constructed to explore OS at a macroscopic level. The abnormally expressed network showed the numerous incorrect data linkages that are present when OS emerges, making it useful as a map of the faults in OS. In theory, the correction of these errors could lead to the prevention and even cure of the disease. Unlike studies in which cancer networks have been formed based purely on gene data, the present study focused on genes and miRNAs, as well as the associations among them, to form the regulatory networks of OS. The constructed regulatory networks were shown to contain numerous self-adaptation associations, which may aid in the analysis of the pathogenesis of OS. By comparing and analyzing the similarities and differences, a number of important pathways were highlighted. A notable finding was the predicted TFs obtained by the P-Match method, which could be used to further study the pathogenesis of OS. In the present study, the mechanism of OS has been systematically analyzed and a theoretical foundation for the
\end{abstract}

Correspondence to: Professor Zhiwen Xu, College of Computer Science and Technology, Jilin University, 2699 Qianjin Street, Changchun, Jilin 130012, P.R. China

E-mail:xuzw@jlu.edu.cn

Abbreviations: OS, osteosarcoma; miRNA, microRNA; TFs, transcription factors; NCBI, National Center for Biotechnology Information; TFBSs, transcription factor binding sites

Key words: osteosarcoma, gene, microRNA, transcription factor, network, pathway mechanism has been provided, which may assist the development of gene therapy targeting OS.

\section{Introduction}

Osteosarcoma (OS) is the most common primary bone malignancy in children and young adults. Mortalities due to malignant neoplasms of the bone and joint represent $8.9 \%$ of all childhood and adolescent cancer mortalities (1).

Transcription factors (TFs) and microRNAs (miRNAs) are prominent regulators of gene expression (2). In molecular biology, TFs are proteins that are able to regulate the transcription of genes by the way of promotion or suppression (3). TFs perform this regulatory function either by binding to the upstream regions of genes alone or through coregulation with other proteins. miRNAs are endogenous, non-coding RNA molecules, which regulate gene expression at a transcriptional level (4). miRNAs play a significant role in numerous biological functions and have been shown to control key cellular processes, including proliferation and apoptosis (5). Several miRNAs have been found to have links with various types of cancer, and miRNAs often exhibit aberrant expression in cancer $(6,7)$.

The genes in which miRNAs are located are known as host genes. The expression of the majority of miRNAs is correlated with the expression of their host genes (8). Rodriguez et al (9) demonstrated that miRNAs are transcribed in parallel with their host transcripts and that the two different transcription classes of miRNA (exonic and intronic) that have been identified may require slightly different mechanisms of biogenesis.

The genes that are targeted by miRNAs are referred to as target genes (targets). A number of databases supply sufficient information to enable the association between the miRNAs and their targets to be studied. Several transcription-profiling studies of miRNA transfection experiments have shown that miRNAs exert a widespread impact on the regulation of their targets (10-13).

A number of experiments in the field of OS have been conducted, leading to the theory that the cancer is caused by abnormally expressed genes and miRNAs; however, the majority of these experiments were conduced from a specific angle (gene or miRNA), which limited the understanding of the pathogenesis of OS from a broader perspective (14-19). The aim of the present study was to focus on all the elements (genes, miRNAs and TFs), instead of focusing on one or 
several of them. Three types of experimentally validated associations exist between the elements in OS, including miRNAs and their host genes, TFs and miRNAs, and miRNAs and the corresponding targets. In the present study, three regulatory networks consisting of an abnormally expressed network, a related network and a global network, were constructed in order to identify the mechanisms in OS and provide a foundation for further research into the condition.

\section{Materials and methods}

Material collection and data processing. The host genes of the human miRNAs were extracted from the National Center for Biotechnology Information (NCBI) and miRBase (20). The experimentally validated dataset of human TFs and the miRNAs that were regulated by them were extracted from TransmiR (21). The experimentally validated dataset of human miRNAs and their targets were extracted from miRTarBase (22) and Tarbase 5.0 (23). The official symbol from the NCBI database, which can be accessed at http://www.ncbi.nlm.nih.gov/gene/, was used to represent all miRNAs and genes.

The abnormally expressed genes of OS were collected from the NCBI single nucleotide polymorphism database (http://www.ncbi.nlm.nih.gov/snp/), the Cancer Genetics Web (http://www.cancerindex.org/geneweb/) and the relevant literature. The related genes were collected from the GeneCards database (24) and relevant literature. In addition, the predicted TFs obtained by the P-Match method were considered to be related genes. Only the TFs that appeared in TransmiR were focused on. The P-Match method is a tool to identify transcription factor binding sites (TFBSs) in 1,000-nucleotide (nt) promoter-region sequences and to map TFBSs onto the promoter region of targets. P-Match combines pattern-matching and weight-matrix approaches in order to provide a higher accuracy of recognition than each of the methods alone (25). The 1,000-nt promoter region sequences of the targets of abnormally expressed miRNAs were downloaded from the University of California Santa Cruz database (26). Since P-Match uses the matrix library as well as sets of aligned known TFBSs collected in Transfac ${ }^{\circledR}$, it facilitates a search for a large variety of different TFBSs (25).

The abnormally expressed miRNAs were collected from mir2Disease (27) and the relevant literature. The OS-related miRNAs were also collected from the relevant literature.

Construction of the three networks. In this study, three regulatory networks of OS (abnormally expressed, related and global) were constructed. The abnormally expressed elements (genes and miRNAs) and the associations between them were extracted to derive the abnormally expressed network. The abnormally expressed network shows a number of the critical data linkages in the progression of OS and acts as a core network.

In order to construct the related network, the related elements (genes and miRNAs) involved the pathogenesis of OS, as well as the associations between them, were extracted. Four subnets from the related network were selected to further elucidate the pathogenic mechanism of OS. The abnormally expressed network was included in the related network.
All of the regulatory associations in OS, with regard to the host genes, miRNAs, targets and TFs, were subsequently extracted to form the global network, which is a regulatory network that incorporates the abnormally expressed and related networks.

\section{Results}

Abnormally expressed network of OS. Fig. 1 shows numerous crucial regulatory pathways and significant elements in OS. This network is composed of 3 TFs, 13 targets, 34 miRNAs and 34 host genes. All the TFs are part of the targets. All nodes are abnormally expressed, with the exception of the host genes. The main focus in Fig. 1 is on the parts of the network that are closely related. The single nodes that do not exhibit regulatory associations with other elements, such as S100A4 and NRG1, have been omitted; however, the ignored elements and pathways also have an effect on the development of OS.

The 3 TF-related pathways are the most significant in Fig. 1. As can be seen from the network, 6 miRNAs target MYC, which regulates 12 miRNAs; 2 miRNAs target MYCN, which regulates 8 miRNAs; and 2 miRNAs target TP53, which regulates 8 miRNAs. Certain data linkages exhibit special features. TP53 and MYC, for example, regulate hsa-miR-29a and hsa-miR-34a, which target MYCN; therefore, it is suggested that MYC and TP53 are able to indirectly affect the expression of MYCN via hsa-miR-29a and hsa-miR-34a. A number of regulatory circuits can also be found in this network. For example, hsa-miR-20a targets MYC and, in turn, hsa-miR-20a is regulated by MYC, suggesting a self-adaptation association between the two. Notably, hsa-miR-24 targets MYC, CDK4 and CDKN2A but is not regulated by any genes, suggesting that it may be the first element to act in the abnormally expressed network. Similarly, MMP2, CDH1, RB1, RUNX2, CDK4, KRAS, CD44, ERBB2, CDKN2A and CTNNB1 are targeted by certain miRNAs but do not regulate any miRNAs, which suggests that they may be the last active elements in the abnormally expressed network. These elements play a critical role in cancer. For example, KRAS is a typical proto-oncogene, which is implicated in various malignancies, including lung adenocarcinoma, mucinous adenoma, ductal carcinoma of the pancreas and colorectal carcinoma (28-34).

The host genes and their miRNAs exhibit certain specific characteristics in the abnormally expressed network. The host genes are considered as abnormally expressed genes due to the abnormal expression of their miRNAs. Fig. 1 shows that a host gene may include one or several miRNAs that are alone or together regulated by specific genes. MIR17HG contains 6 miRNAs (hsa-miR-18a, hsa-miR-19a, hsa-miR-19b-1, hsa-miR-20a, hsa-miR-92a-1 and hsa-miR-17), which are regulated by MYC and MYCN. Among the 6 miRNAs, hsa-miR-17 and hsa-miR-20a separately form self-adaptation associations with MYC. Furthermore, a single miRNA may be located in several genes; hsa-miR-191, for example, is present in DALRD3 and NDUFAF3.

Fig. 1 shows certain significant characteristics of the abnormally expressed genes and miRNAs in OS. In theory, the adjustment of the abnormally expressed data to a normal level could enable the control of the OS. The abnormally 


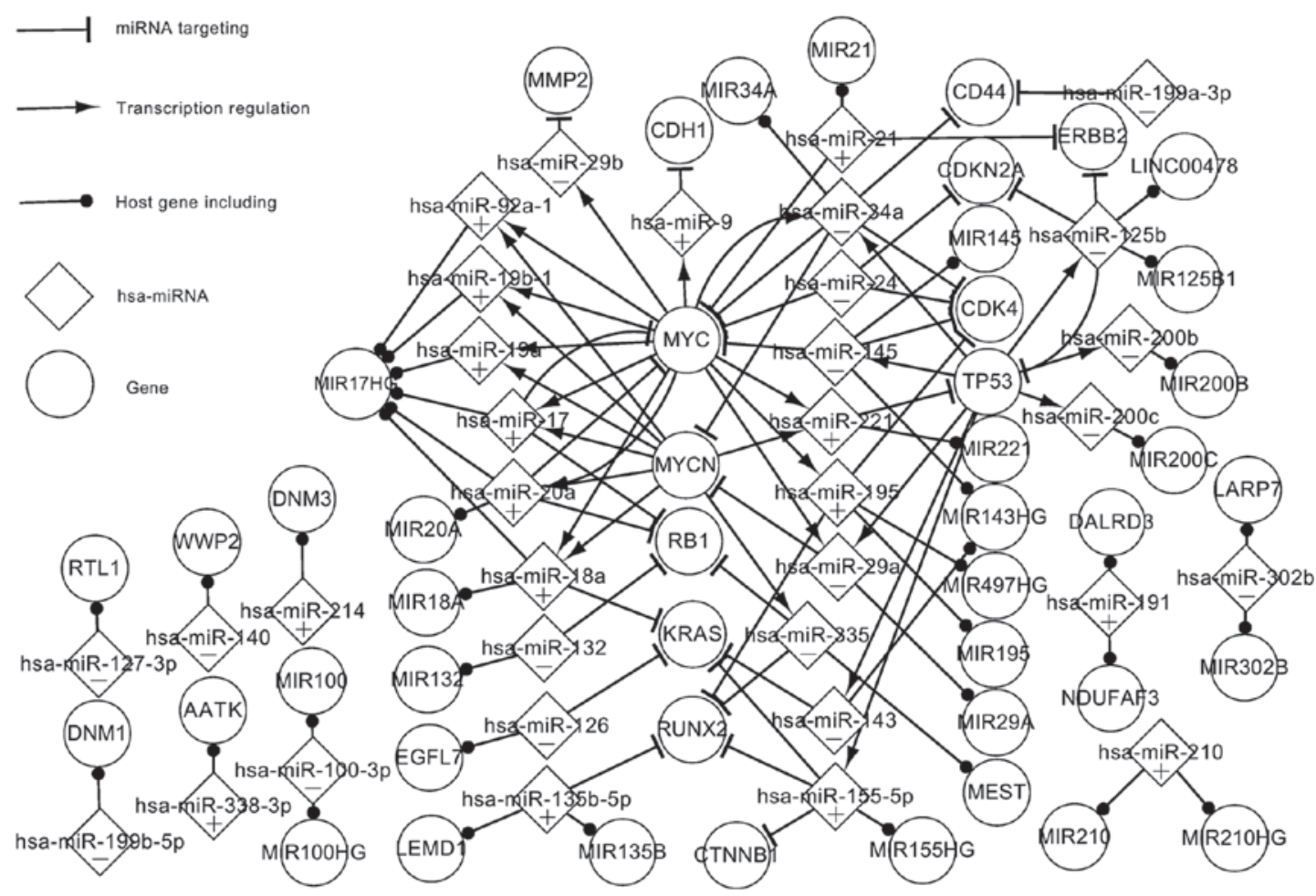

Figure 1. Abnormally expressed network of the genes and miRNAs in osteosarcoma. The ' + ' and '-' symbols show how the expression level of the abnormally expressed miRNA compares with the expression level in the normal tissue. '+' represents upregulated, overexpressed, elevated and increased; '-' represents downregulated, reduced, under-expressed, lost and decreased. miRNA, microRNA.

expressed network partially elucidates the pathogenesis of OS.

Related network of OS. The abnormally expressed network is contained within the related network. In addition to the abnormally expressed data, numerous related elements are shown. The network contains information on an increased number of regulatory associations among the genes and miRNAs. Four subnets were selected from the related network in order to describe the network more clearly. Fig. 2A centers on the association between hsa-miRNA-125b and other elements. As an abnormally expressed miRNA, hsa-miRNA-125b is regulated by an abnormally expressed TF (TP53) and a related TF (NFKB1), while hsa-miRNA-125b targets 8 genes (CDKN2A, TP53, GLI1, CYP24A1, IGF2, HMGA2, VDR and ERBB2). Among these genes, CDKN2A, TP53 and ERBB2 are abnormally expressed genes, whereas the others are not. TP53 and hsa-miRNA-125b form a self-adaptation association. Fig. 2B is composed of $1 \mathrm{TF}, 3$ targets and 1 miRNA. It can be seen that TCF3, which is a related TF, regulates hsa-miRNA-451, targeting 3 genes (MMP2, MMP9 and ABCB1). Among these genes, MMP2 is an abnormally expressed gene and the others are not. Fig. $2 \mathrm{C}$ is a subnet around the regulatory associations between HIF1A and other elements. As can be seen from Fig. 2C, HIF1A regulates hsa-miR-210 and hsa-miR-424; hsa-miR-210 targets E2F3 and hsa-miR-424 targets ATF6. Fig. 2D shows MYC, which regulates 17 miRNAs, which, in turn, target 36 genes. Three miRNA-MYC pairs form a self-adaptation association in Fig. 2D: hsa-miR-20a and MYC, hsa-miR-17 and MYC, and hsa-miR-34a and MYC. In addition, hsa-miR-106b, hsa-miR-17, hsa-miR-20a and hsa-miR-93 target CDKN1A and E2F1; hsa-miR-106b, hsa-miR-17 and hsa-miR-20a target RB1 and RBL2; and hsa-miR-17, hsa-miR-20a and hsa-miR-92a-1 target THBS1. Fig. 2 demonstrates that the abnormally expressed and related factors interact with each other to affect the OS network. The related network expands the additional topological associations of the abnormally expressed elements and partially elucidates the mechanism of OS.

Global network of OS. The global network includes the abnormally expressed and related networks. All the elements and associations of the global network have been experimentally validated. Due to the complexity and size of the network, it is difficult to provide a clear description of the findings; therefore, the network will not be described in detail but will instead be utilized as a tool throughout the remainder of the study.

Transcriptional network of predicted TFs. Predicted TFs are TFs that are believed to be involved in the transcriptional processes of OS. Fig. 3 depicts the regulatory interactions between these predicted TFs and the abnormally expressed miRNAs, in addition to the targets, in OS. These factors influence their successors by regulating or targeting them in the transcriptional network. As shown in Fig. 3, NFKB1, E2F1, E2F3 and ZEB1 are more significant than the other TFs. Specifically, NFKB1, E2F3 and ZEB1 coregulate hsa-miR-34a, which targets E2F1, CDK4, CD44, MYC, E2F3 and MYCN. E2F3 and hsa-miR-34a form a self-adaptation association. As can be observed, NFKB1 regulates 10 miRNAs (hsa-miR-125b, hsa-miR-146a-5p, hsa-miR-155-5p, hsa-miR-21, hsa-miR-214, hsa-miR-29a, 


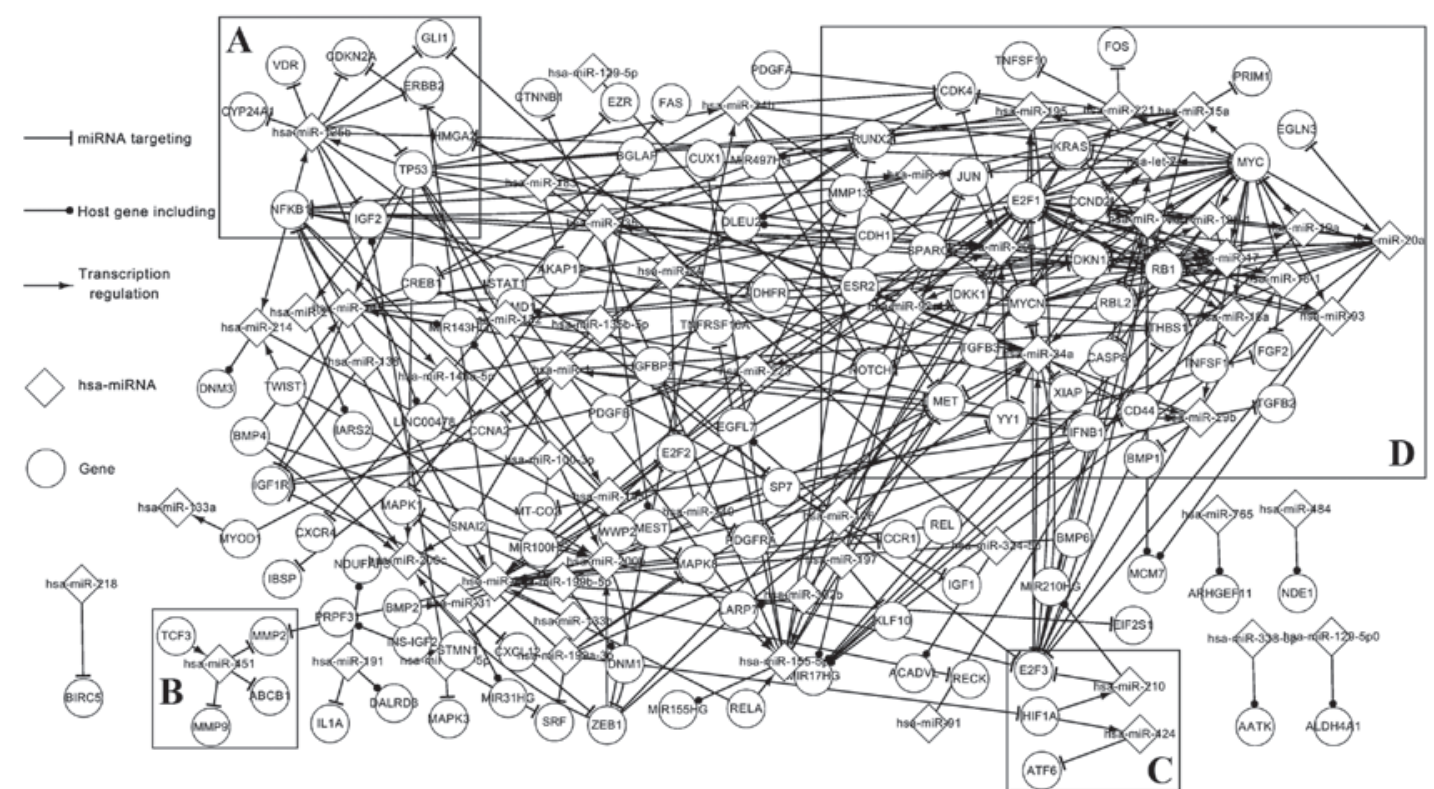

A

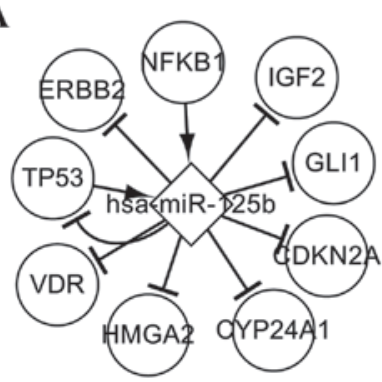

B

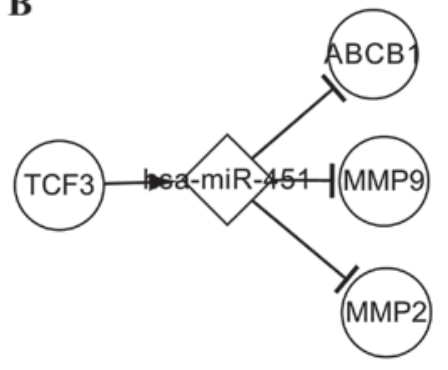

C

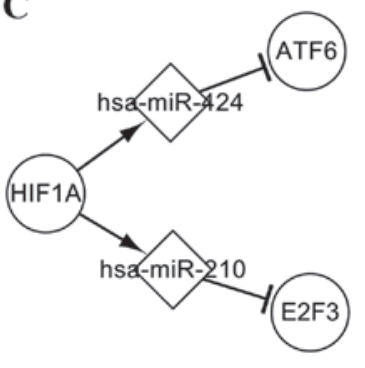

D

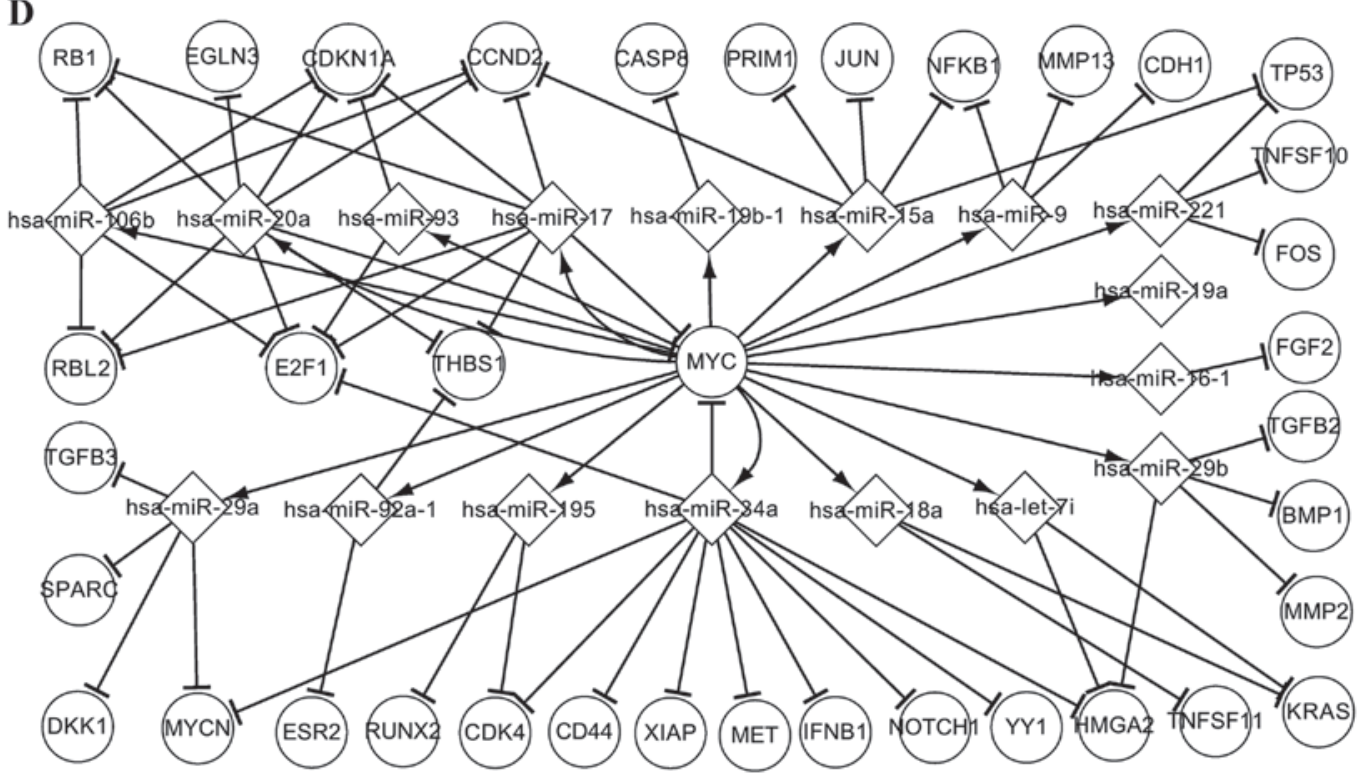

Figure 2. Related network of the genes and miRNAs in osteosarcoma, including four subnets. Each subnet concerns a specific element and the regulatory associations between that element and other elements: (A) hsa-miRNA-125b, (B) hsa-miRNA-451, (C) HF1A and (D) MYC. miRNA, microRNA.

hsa-miR-29b, hsa-miR-34a, hsa-miR-9 and hsa-miR-17) and is targeted by 3 miRNAs (hsa-miR-146a-5p, hsa-miR-21 and hsa-miR-9). These 3 miRNAs separately form self-adaptation associations with NFKB1. The other TFs are similar to NFKB1. In Fig. 3, it can be observed that a single TF may regulate one or several abnormally expressed miRNAs, and an abnormally expressed miRNA may target one or several TFs. A TF indirectly affects other TFs via specific abnormally expressed miRNAs, and an abnormally expressed miRNA indirectly influences other miRNAs via certain TFs. The predicted TFs may further reveal the transcriptional mechanism associated with OS. 


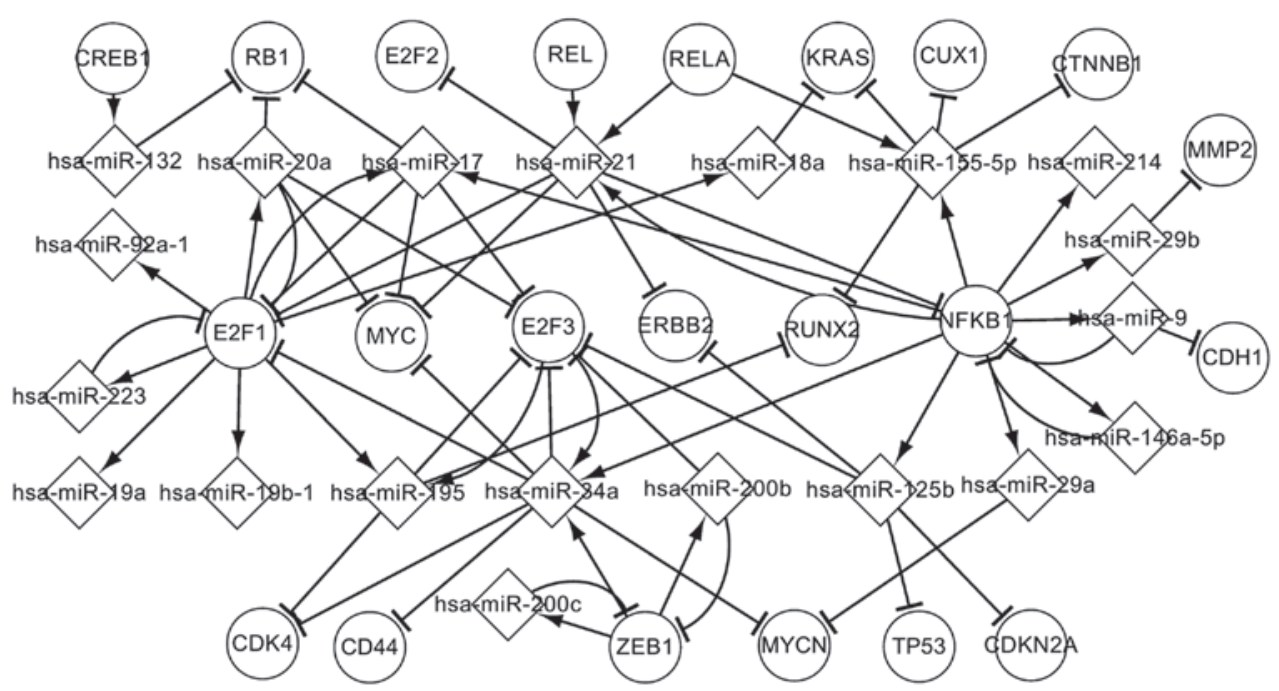

Figure 3. Transcriptional network of predicted transcription factors and abnormally expressed miRNAs, as well as target genes, in osteosarcoma. miRNA, microRNA.
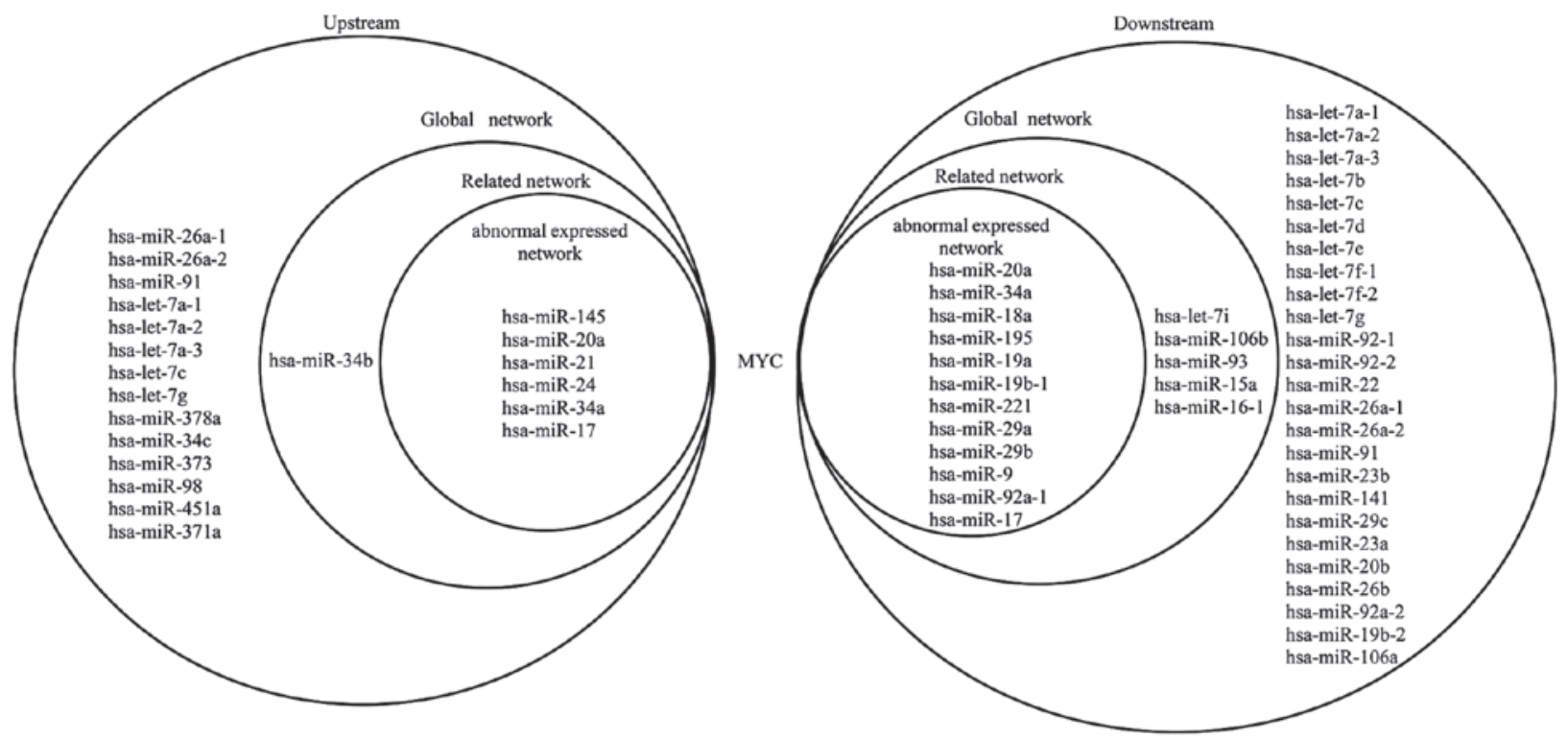

Figure 4. Regulatory associations between miRNAs and MYC. 'Upstream' shows certain miRNAs that target MYC in the three networks, and 'Downstream' refers to miRNAs that are regulated by MYC in the three networks. miRNA, microRNA.

Regulatory associations involving abnormally expressed genes. The upstream and downstream information of the predicted TFs and data on the abnormally expressed genes and miRNAs were collected to describe the network of OS more clearly. The abnormally expressed genes were initially concentrated on. 'Upstream' refers to miRNAs that target abnormally expressed genes, and 'downstream' refers to miRNAs that are regulated by abnormally expressed genes in the three networks. The abnormally expressed genes were divided into three classes by extracting and classifying all the regulatory associations involving the abnormally expressed genes in the three networks. The first class of gene included genes with only upstream, but no downstream, elements, such as CD44. CD44 is targeted by a number of miRNAs in the three networks but does not regulate any miRNAs. The second class of gene included genes with only downstream, but no upstream, elements, such as CDKN2A. CDKN2A regulates hsa-miR-410 in the global network but is not targeted by any miRNAs. The third class of gene had both upstream and downstream elements. MYC is considered as an example and is discussed in the following section.

Fig. 4 shows the upstream and downstream elements of MYC in the three networks. The figure shows that 6 miRNAs target MYC and MYC regulates 12 miRNAs in the abnormally expressed network; 7 miRNAs target MYC and MYC regulates 17 miRNAs in the related network; and 21 miRNAs target MYC and MYC regulates 42 miRNAs in the global network. The elements upstream of MYC indirectly affect the elements downstream of MYC. hsa-miR-20a, hsa-miR-34a and hsa-miR-17 target MYC and are regulated by MYC in return, which forms a self-adaptation association in the three networks. Furthermore, MYC indirectly affects the expression 


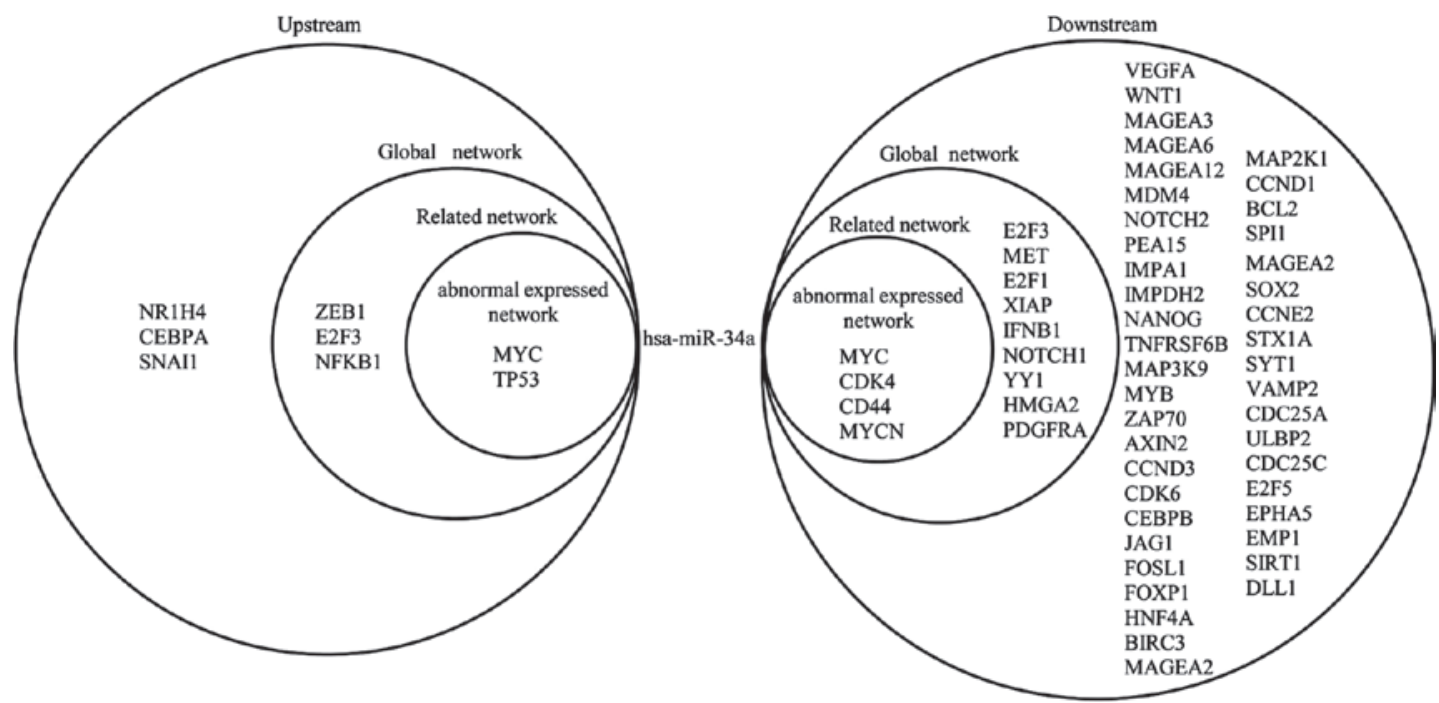

Figure 5. Regulatory associations between hsa-miR-34a and genes. 'Upstream' shows certain genes that regulate hsa-miR-34a in the three networks, and 'Downstream' shows targets of hsa-miR-34a in the three networks.
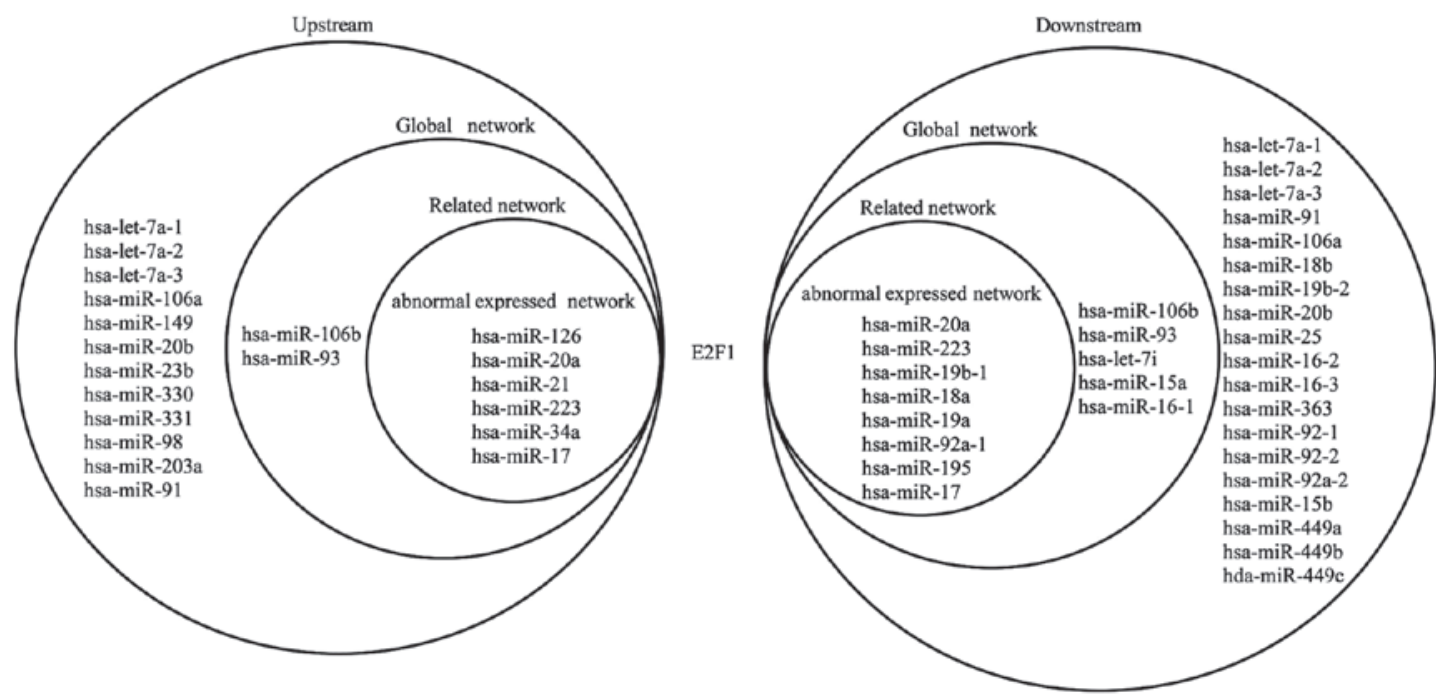

Figure 6. Regulatory associations between E2F1 and miRNAs. 'Upstream' shows certain miRNAs that target E2F1 in the three networks, and 'Downstream' shows miRNAs that are regulated by E2F1 in the three networks. miRNA, microRNA.

of other genes via a number of miRNAs. For example, MYC regulates hsa-miR-20a, which targets RB1. Certain genes can also indirectly influence MYC through specific miRNAs. For example, TP53 regulates hsa-miR-145, which targets MYC in turn. Other genes are similar to MYC.

Regulatory associations involving abnormally expressed miRNAs. Similar to the abnormally expressed genes, the upstream and downstream information of abnormally expressed miRNAs was extracted. The upstream data show genes that regulate abnormally expressed miRNAs, and the downstream data show targets of the abnormally expressed miRNAs in the three networks. The abnormally expressed miRNAs were divided into two classes: i) Downstream elements only (no upstream elements), for example hsa-miR-376c, which targets ACVR1C in the global network and is not regulated by any genes; ii) both upstream and downstream elements. The subsequent experiments focused on hsa-miR-34a.
Fig. 5 shows the upstream and downstream elements of hsa-miR-34a in the three networks. In the abnormally expressed network, 2 genes regulate hsa-miR-34a, which, in turn, targets 4 genes. In the related network, 5 genes regulate hsa-miR-34a, which, in turn, targets 13 genes. In the global network, 8 genes regulate hsa-miR-34a, which targets 56 genes. As can be noted from Fig. 5, MYC regulates hsa-miR-34a and hsa-miR-34a targets MYC in return, which forms a self-adaptation association in the three networks. In addition, E2F3 regulates hsa-miR-34a and hsa-miR-34a targets E2F3 in return, which forms a self-adaptation association in the related and global networks. In addition to this, hsa-miR-34a indirectly affects other miRNAs via certain genes: hsa-miR-34a, for example, targets MYC, which regulates hsa-miR-17. A number of miRNAs also indirectly influence hsa-miR-34a by targeting specific genes. For example, hsa-miR-221 targets TP53, but regulates hsa-miR-34a. 
Regulatory associations involving predicted TFs. The same method was applied to the predicted TFs. The upstream data show miRNAs that target the predicted TFs in the three networks and the downstream data show miRNAs that are regulated by the predicted TFs in the three networks. The predicted TFs were divided into three classes. The first class of predicted TFs included TFs with only upstream, not downstream, elements, such as STAT1. STAT1 is targeted by hsa-miR-145 in the three networks but does not regulate any miRNAs. By contrast, the second class of predicted TFs included TFs with no upstream, only downstream, elements, such as REL. REL regulates hsa-miR-21 in the three networks but is not targeted by any miRNAs. The third class included TFs with both upstream and downstream elements; E2F1 is taken as an example.

Fig. 6 shows the upstream and downstream elements of E2F1 in the three networks. Six miRNAs are shown to target E2F1, which regulates 8 miRNAs in the abnormally expressed network; 8 miRNAs target E2F1, which regulates 13 miRNAs in the related network; and 20 miRNAs target E2F1, which regulates 32 miRNAs in the global network. It can also be noted that hsa-miR-106b and hsa-miR-93 separately form self-adaptation associations with $\mathrm{E} 2 \mathrm{~F} 1$ in the related and global networks. hsa-miR-20a, hsa-miR-17 and hsa-miR-223 separately form self-adaptation associations with E2F1 in the three networks. E2F1 is not abnormally expressed in OS, but the 3 miRNAs are abnormally expressed; therefore, these 3 miRNAs may indirectly lead to the aberrant expression of other miRNAs via E2F1.

\section{Discussion}

By analyzing the current data associated with OS, three regulatory networks at different levels (the abnormally expressed, related and global networks) were derived. This study mainly focused on the abnormally expressed network in comparison with the related and global networks. A number of significant regulatory associations involving the three types of elements (abnormally expressed genes, abnormally expressed miRNAs and TFs) were found in the abnormally expressed network. Certain data linkages that exert key biological functions in OS were highlighted in the abnormally expressed network, such as MYC-hsa-miR-34a-MYC-hsa-miR-335-RUNX2. These data linkages of elements mutated in OS are critical to the development of an enhanced understanding of the molecular pathogenesis of this disease.

Certain data linkages are involved in processes associated with other types carcinoma, as well as OS. In breast tumors, for example, TP53 drives invasion through the upregulation of hsa-miR-155 (35), which suggests that the interactions of genes can be expanded from one cancer to another. Furthermore, the abnormal expression of miRNAs and genes is considered to determine the development, metastasis and therapy of OS. The abnormally expressed network constructed in the present study, which is based on experimentally validated data, shows numerous incorrect signaling pathways that occur in the human body when OS emerges; therefore, this network represents a core misregulation network and could be used to treat those patients suffering from OS through the regulation of the expression of abnormal factors to normal levels.
Furthermore, the network could be used in the prevention of OS. The adjustment of the abnormally expressed elements and incorrect linkages formed by these elements to a normal state could, in theory, facilitate the control or even the cure of the cancer.

In the present study, the related network, which contributes to the understanding of numerous processes associated with OS, and the global network, in which all the data have been experimentally validated, were additionally constructed. Particularly notable are the TFs predicted from the P-Match method, as they may reveal the pathogenesis of OS. These results and the comprehensive data may lead biologists to the further study of the mechanisms associated with carcinogenicity and the therapy of OS, which may result in improvements in the prognosis, diagnosis and therapy of OS. The present study may provide novel insights into cancer biology.

\section{Acknowledgements}

This study was supported by the Science and Technology Development Plan of Jilin Province (grant no. 20130101166JC).

\section{References}

1. Ottaviani G and Jaffe N: The epidemiology of osteosarcoma. Cancer Treat Res 152: 3-13, 2009.

2. Hobert O: Gene regulation by transcription factors and microRNAs. Science 319: 1785-1786, 2008.

3. Tran DH, Satou K, Ho TB and Pham TH: Computational discovery of miR-TF regulatory modules in human genome. Bioinformation 4: 371-377, 2010.

4. Yue J and Tigyi G: MicroRNA trafficking and human cancer. Cancer Biol Ther 5: 573-578, 2006.

5. Bartel DP: MicroRNAs: Genomics, biogenesis, mechanism, and function. Cell 116: 281-297, 2004.

6. McManus MT: MicroRNAs and cancer. Semin Cancer Biol 13: 253-258, 2003.

7. Volinia S, Calin GA, Liu CG, Ambs S, Cimmino A, Petrocca F, Visone R, Iorio M, Roldo C, Ferracin M, et al: A microRNA expression signature of human solid tumors defines cancer gene targets. Proc Natl Acad Sci USA 103: 2257-2261, 2006.

8. Baskerville S and Bartel DP: Microarray profiling of microRNAs reveals frequent coexpression with neighboring miRNAs and host genes. RNA 11: 241-247, 2005.

9. Rodriguez A, Griffiths-Jones S, Ashurst JL and Bradley A: Identification of mammalian microRNA host genes and transcription units. Genome Res 14: 1902-1910, 2004.

10. Naeem H, Küffner R and Zimmer R: MIRTFnet: Analysis of miRNA regulated transcription factors. PLoS One 6: e22519, 2011.

11. Selbach M, Schwanhäusser B, Thierfelder N, Fang Z, Khanin R and Rajewsky N: Widespread changes in protein synthesis induced by microRNAs. Nature 455: 58-63, 2008.

12. Lim LP, Lau NC, Garrett-Engele P, Grimson A, Schelter JM, Castle J, Bartel DP, Linsley PS and Johnson JM: Microarray analysis shows that some microRNAs downregulate large numbers of target mRNAs. Nature 433: 769-773, 2005.

13. Huang JC, Babak T, Corson TW, Chua G, Khan S, Gallie BL, Hughes TR, Blencowe BJ, Frey BJ and Morris QD: Using expression profiling data to identify human microRNA targets. Nat Methods 4: 1045-1049, 2007.

14. Sztán M, Pápai Z, Szendrôi M, Looij M and Oláh E: Allelic losses from chromosome 17 in human osteosarcomas. Pathol Oncol Res 3: 115-120, 1997.

15. Choy E,HornicekF,MacConaillL,Harmon D, TariqZ, Garraway L and Duan Z: High-throughput genotyping in osteosarcoma identifies multiple mutations in phosphoinositide-3-kinase and other oncogenes. Cancer 118: 2905-2914, 2012.

16. Kansara M and Thomas DM: Molecular pathogenesis of osteosarcoma. DNA Cell Biol 26: 1-18, 2007.

17. Hong Q, Fang J, Pang Y and Zheng J: Prognostic value of the microRNA-29 family in patients with primary osteosarcomas. Med Oncol 31: 37, 2014. 
18. Wang Z, Cai H, Lin L, Tang M and Cai H: Upregulated expression of microRNA-214 is linked to tumor progression and adverse prognosis in pediatric osteosarcoma. Pediatr Blood Cancer 61: 206-210, 2014.

19. Lulla RR, Costa FF, Bischof JM, Chou PM, de F Bonaldo M Vanin EF and Soares MB: Identification of differentially expressed microRNAs in osteosarcoma. Sarcoma 2011: 732690, 2011.

20. Kozomara A and Griffiths-Jones S: miRBase: Integrating microRNA annotation and deep-sequencing data. Nucleic Acids Res 39: D152-D157, 2011.

21. Wang J, Lu M, Qiu C and Cui Q: TransmiR: A transcription factor-microRNA regulation database. Nucleic Acids Res 38: D119-D122, 2010.

22. Hsu SD, Lin FM, Wu WY, Liang C, Huang WC, Chan WL, Tsai WT, Chen GZ, Lee CJ, Chiu CM, et al: miRTarBase: A database curates experimentally validated microRNA-target interactions. Nucleic Acids Res 39: D163-D169, 2011.

23. Papadopoulos GL, Reczko M, Simossis VA, Sethupathy P and Hatzigeorgiou AG: The database of experimentally supported targets: A functional update of TarBase. Nucleic Acids Res 37: D155-D158, 2009

24. Safran M, Dalah I, Alexander J, Rosen N, Iny Stein T, Shmoish M, Nativ N, Bahir I, Doniger T, Krug H, et al: GeneCards Version 3: The human gene integrator. Database (Oxford) 2010: baq020, 2010

25. Chekmenev DS, Haid C and Kel AE: P-Match: Transcription factor binding site search by combining patterns and weight matrices. Nucleic Acids Res 33: W432-W437, 2005.

26. Fujita PA, Rhead B, Zweig AS, Hinrichs AS, Karolchik D, Cline MS, Goldman M, Barber GP, Clawson H, Coelho A, et al: The UCSC genome browser database: Update 2011. Nucleic Acids Res 39: D876-D882, 2011.

27. Jiang Q, Wang Y, Hao Y, Juan L, Teng M, Zhang X, Li M, Wang $G$ and Liu Y: miR2Disease: A manually curated database for microRNA deregulation in human disease. Nucleic Acids Res 37: D98-D104, 2009.
28. Kranenburg O: The KRAS oncogene: Past, present, and future. Biochim Biophys Acta 1756: 81-82, 2005.

29. Marks JL, Broderick S, Zhou Q, Chitale D, Li AR, Zakowski MF, Kris MG, Rusch VW, Azzoli CG, Seshan VE, et al: Prognostic and therapeutic implications of EGFR and KRAS mutations in resected lung adenocarcinoma. J Thorac Oncol 3: 111-116, 2008.

30. Suda K, Tomizawa K and Mitsudomi T: Biological and clinical significance of KRAS mutations in lung cancer: an oncogenic driver that contrasts with EGFR mutation. Cancer Metastasis Rev 29: 49-60, 2010.

31. Hershkovitz D, Vlodavsky E, Simon E and Ben-Izhak O: KRAS mutation positive mucinous adenocarcinoma originating in mature ovarian teratoma: Case report and review of literature. Pathol Int 63: 611-614, 2013.

32. Yatsuoka T, Sunamura M, Furukawa T, Fukushige S, Yokoyama T, Inoue H, Shibuya K, Takeda K, Matsuno S and Horii A: Association of poor prognosis with loss of $12 \mathrm{q}, 17 \mathrm{p}$, and $18 \mathrm{q}$, and concordant loss of $6 \mathrm{q} / 17 \mathrm{p}$ and $12 \mathrm{q} / 18 \mathrm{q}$ in human pancreatic ductal adenocarcinoma. Am J Gastroenterol 95: 2080-2085, 2000.

33. Abubaker J, Bavi P, Al-Haqawi W, Sultana M, Al-Harbi S, Al-Sanea N, Abduljabbar A, Ashari LH, Alhomoud S, Al-Dayel F, et al: Prognostic significance of alterations in KRAS isoforms KRAS-4A/4B and KRAS mutations in colorectal carcinoma. J Pathol 219: 435-445, 2009.

34. Smakman N, Borel Rinkes IH, Voest EE and Kranenburg O: Control of colorectal metastasis formation by K-Ras. Biochim Biophys Acta 1756: 103-114, 2005.

35. Neilsen PM, Noll JE, Mattiske S, Bracken CP, Gregory PA, Schulz RB, Lim SP, Kumar R, Suetani RJ, Goodall GJ and Callen DF: Mutant p53 drives invasion in breast tumors through up-regulation of miR-155. Oncogene 32: 2992-3000, 2013. 